Décadrages Décadrages

cinéma, à travers champs Cinéma, à travers champs

$20 \mid 2012$

Peter Watkins

\title{
Entretien avec Olivier Père
}

\section{Carine Bernasconi}

\section{(2) OpenEdition}

Journals

Édition électronique

URL : https://journals.openedition.org/decadrages/263

DOI : $10.4000 /$ decadrages. 263

ISSN : 2297-5977

\section{Éditeur}

Association Décadrages

\section{Édition imprimée}

Date de publication : 10 avril 2012

Pagination : 123-130

ISBN : 978-2-9700668-4-2

ISSN : 2235-7823

Référence électronique

Carine Bernasconi, «Entretien avec Olivier Père », Décadrages [En ligne], 20 | 2012, mis en ligne le 10 avril 2013, consulté le 03 avril 2022. URL : http://journals.openedition.org/decadrages/263 ; DOI : https://doi.org/10.4000/decadrages.263

(B) Décadrages 


\section{Entretien avec Olivier Père}

\section{par Carine Bernasconi}

Avec l'arrivée en 2010 du Français Olivier Père à la direction artistique du Festival de Locarno 1 - auparavant programmateur à la Cinémathèque française, délégué général de la Quinzaine des réalisateurs à Cannes (de 2004 à 2009), critique de films pour plusieurs revues spécialisées -, c'est un îlot de la cinéphilie parisienne qui s'est déplacé dans le canton du Tessin. En effet, à travers la figure de son directeur artistique, le festival tisse des liens étroits avec la cinéphilie, notamment dans la façon de penser et de justifier les choix de programmation.

Cet entretien, qui s'inscrit dans la recherche que nous menons sur les festivals ${ }^{2}$, a été l'occasion de discuter la façon dont la cinéphilie a informé ses héritiers, c'est-à-dire comment les idées, les concepts et les représentations associés à cette notion et qui ont participé à son essor continuent d'influencer ses descendants et, en l'occurrence, la ligne éditoriale du Festival de Locarno.
1 Olivier Père, actuel directeur artistique du Festival de Locarno, en a dirigé les éditions 2010 et 2011. Cet entretien a été réalisé en mars 2011 après une seule édition sous sa direction.

2 Dans le numéro 19 de Décadrages (automne 2011), Charlotte Bouchez et Nicolas Brulhart consacrent une étude à l'histoire du FIFF: "Faire I'histoire du FIFF, questions de méthode pour un objet instable", ainsi qu'un entretien avec Martial Knaebel, I'un de ses membres fondateurs.

\section{Vous êtes présenté dans les médias comme un cinéphile,} qu'est-ce que cela évoque pour vous?

Je viens de la cinéphilie, car la cinéphilie est une culture de contrebande: c'est tout ce qu'on n'étudie pas à l'université ou à l'école, c'est la culture qu'on se forge soi-même en dehors de tout cursus universitaire. Je n’ai jamais étudié le cinéma mais, parallèlement à ma scolarité et à mes études, j'en ai développé une véritable passion: je fais peut-être partie de la dernière génération qui a développé cette passion par l'intermédiaire des salles. Aujourd'hui, les passionnés voient les films par d'autres biais comme le DVD ou internet. Les salles de cinéma ont peut-être perdu ce rôle. Pour moi, le fait de me rendre dans une salle de quartier où je passais mes mercredis après-midi et mes samedis après-midi signifiait la possibilité de m'évader dans un lieu qui ne soit ni l'école ni le foyer parental.

\section{De quelle manière s'est développée votre cinéphilie? Et dans quels lieux?}

J'ai passé mon enfance et mon adolescence dans une ville de province, Saint-Etienne. Issu d'un milieu petit bourgeois, je ne viens pas d'une famille particulièrement cultivée, ni très ouverte aux arts: je voyais les films de Walt Disney comme tout le monde, mais dès l'enfance j’ai été attiré par le cinéma qui représentait un moyen d'évasion et une ouverture sur le monde extérieur, sur l'étranger, qui stimulait mon imaginaire d'enfant. J'ai vite développé une fascination et j’ai commencé à y aller avec ma mère, puis seul. Je voyais les films d'aventures et de science-fiction du début des années 1980, ainsi que les James Bond, Star Wars et Flash Gordon, essentiellement des films de genre. La chance que j’ai eue est qu'à Saint-Etienne, ville ouvrière de tradition communiste, il y avait des maisons de la culture et des salles municipales art et essai qui proposaient des films de répertoire, des films de patrimoine 
ainsi que des films plus indépendants. Je pouvais donc voir les grosses productions américaines de Scorsese, Coppola, De Palma ou Spielberg en version française, mais également des films plus anciens en version originale sous-titrée, connotés artetessai, comme ceux de Jarmush par exemple. C'est donc à Saint-Etienne que j'ai vu au cinéma les intégrales de Renoir, Visconti, Fuller ou Tarkovski. A seize ans, j’avais déjà une bonne connaissance du cinéma : je lisais beaucoup de livres, de revues, je découpais des articles, de manière compulsive et obsessionnelle. Comme il était difficile en province d'avoir accès au cercle des cinéphiles, je réussissais à entraîner avec moi certains de mes petits camarades: plusieurs sont devenus cinéphiles sous mon influence... Il y avait déjà cette idée de faire partager cette passion qui allait devenir mon métier.

\section{Quels ont été à cette époque les cinéastes et les films qui vous ont marqué?}

Quand j'étais enfant, Blade Runner (Ridley Scott, USA, 1982) ou Les aventuriers de l'arche perdue (Steven Spielberg, USA, 1981) ${ }^{3}$, ainsi que les autres films de Spielberg, ont représenté de véritables chocs pour moi. Puis, à l'âge de treize ans, j’ai découvert la cinéphilie avec les films de Brian de Palma: j’ai vu au cours de la même semaine Scarface (USA, 1983) et Phantom of Paradise (USA, 1974) et cela a changé mon rapport au cinéma: j’ai découvert que le cinéma pouvait changer la vie des spectateurs. J'ai été très bouleversé par Elephant Man (David Lynch, USA, 1980) et puis des films plus classiques comme La strada (Federico Fellini, Italie, 1954) et ensuite, vers l'âge de seize, dix-sept

\footnotetext{
3 Le Festival de Locarno a remis, lors de l'édition 2011, un Léopard d'honneur à l'acteur américain Harrison Ford qui tient le rôle principal de Blade Runner ainsi que celui des Aventuriers de l'arche perdu. La remise du prix, qui a eu lieu sur la Piazza Grande avant la projection de Cow Boys and Aliens (Jon Favreau, USA, 2011), a été précédée d'un montage de séquences extraites de la filmographie d'Harrison Ford où figuraient notamment les deux films cités plus haut. Le Léopard d'honneur a été remis par Olivier Père.
}

ans, j’ai découvert le cinéma moderne avec Godard, Pasolini et Kubrick qui ont été, en quelque sorte, mes cinéastes de chevet durant mon adolescence.

\section{Vous avez donc découvert tous ces films en salles?}

Oui, car la vidéo n'était pas encore démocratisée. Un de mes copains avait un magnétoscope, je louais des VHS et on passait des week-ends chez lui quand ses parents n'étaient pas là. Mais, par exemple, Phantom of Paradise n'existait pas en vidéo, alors quand il passait à Saint-Etienne, j'allais le voir le plus de fois possible de peur qu'il ne repasse plus (je pouvais le voir cinq ou sept fois dans la même semaine!). Ces dix dernières années, les choses ont vraiment changé avec le DVD.

\section{Quelles étaient les revues qui nourrissaient alors votre cinéphilie?}

Starfix 4 , que j'ai découverte à l'âge de neuf ou dix ans et qui a accompagné mon adolescence, a été la revue fondatrice de ma cinéphilie: consacrée uniquement au cinéma de genre, un peu comme Mad Movies aujourd'hui, elle était plutôt novatrice pour l'époque avec de bons rédacteurs qui s'intéressaient beaucoup aux films de Cronenberg, De Palma ou Argento avant que ces cinéastes ne soient reconnus par une presse plus sérieuse. Starfix se situait dans la lignée de Métal burlant ${ }^{5}$ ou de $M a d$, inspiré de la contre-culture américaine adaptée à la France. Sa durée fut assez brève mais elle a marqué les esprits car certains de ses rédacteurs, pour la plupart des nerds, sont passés à la réalisation: Christophe Gans par exemple, qui en était le rédacteur en chef, a fait du cinéma ${ }^{6}$. Pour le jeune

4 La revue Starfix, créée par Christophe Gans et Doug Headline, est parue de janvier 1983 à décembre 1990 (90 numéros).

5 Le magazine Métal hurlant était consacré à la bande dessinée de science-fiction.

6 Christophe Gans a, entre autres, réalisé le long métrage Le pacte des loups (France, 2001). 
garçon que j'étais, c'était une ouverture, extrêmement excitante, sur le cinéma et sur ce qui se passait à Paris. Je guettais sa sortie avec anxiété. C’est plus tard que j’ai commencé à lire les Cahiers du cinéma: le premier numéro que j'ai acheté c'était pour la mort de Cassavetes $(1989, n d l r)$. Je lisais également des revues plus commerciales comme Première.

\section{Paris vous attirait déjà comme le lieu où vous pourriez} vivre votre cinéphilie?

Avant de m'installer à Paris, il m'arrivait d'y faire des séjours où je voyais cinq films par jour, tout ce que je rêvais de voir et qui ne passait pas à Saint-Etienne. J'allais dans les salles du quartier Latin: l'Accatone, le Reflet Médicis, les Actions. Je visionnais de nombreux films des années 1970 que je n'avais pas pu voir auparavant: Pasolini, Kubrick, Fellini... Puis, à dixneuf ans, je suis venu à Paris, officiellement pour faire des études de Lettres à la Sorbonne, mais en fait l'intérêt était de voir des films. Je fréquentais alors essentiellement la Cinémathèque : ayant du retard à rattraper, j'allais voir les films anciens. Ma passion pour le cinéma de genre et le cinéma fantastique ne m’ayant pas quitté depuis l'enfance, j’ai assisté à cette époque au déclin des salles spécialisées dans des quartiers populaires comme Pigalle, Strasbourg Saint-Denis et les Grands Boulevards, qui projetaient des films de série $\mathrm{B}$, de série $\mathrm{Z}$, d'horreur, d'arts martiaux ou des films érotiques. Les conditions étaient particulières car les copies étaient en lambeaux et les salles fréquentées par des sans-abris. A Paris, mon éclectisme s'est développé et j'allais voir autant les films d'Andy Wahrol, de Kenneth Anger que ceux de Jess Franco ou de Brian De Palma. Nous formions, à cette époque, une bande et nous allions ensemble au cinéma et à la Cinémathèque.

Au cours de ces années de formation, n’avez-vous pas songé à devenir réalisateur?

J'ai tenté l'INSAS à Bruxelles, je n'ai pas osé me présenter à la FEMIS, j'ai passé les deux premiers examens mais j'ai raté l'oral: ce fut la meilleure chose qui me soit arrivée car j'étais un cinéphile, pas un cinéaste. Les choses étaient confuses car ma passion pour le cinéma était dévorante: je cherchais un moyen de rapprocher le plus possible ma vie professionnelle

Remise du Léopard d'honneur à Harrison Ford sur la Piazza Grande en 2011

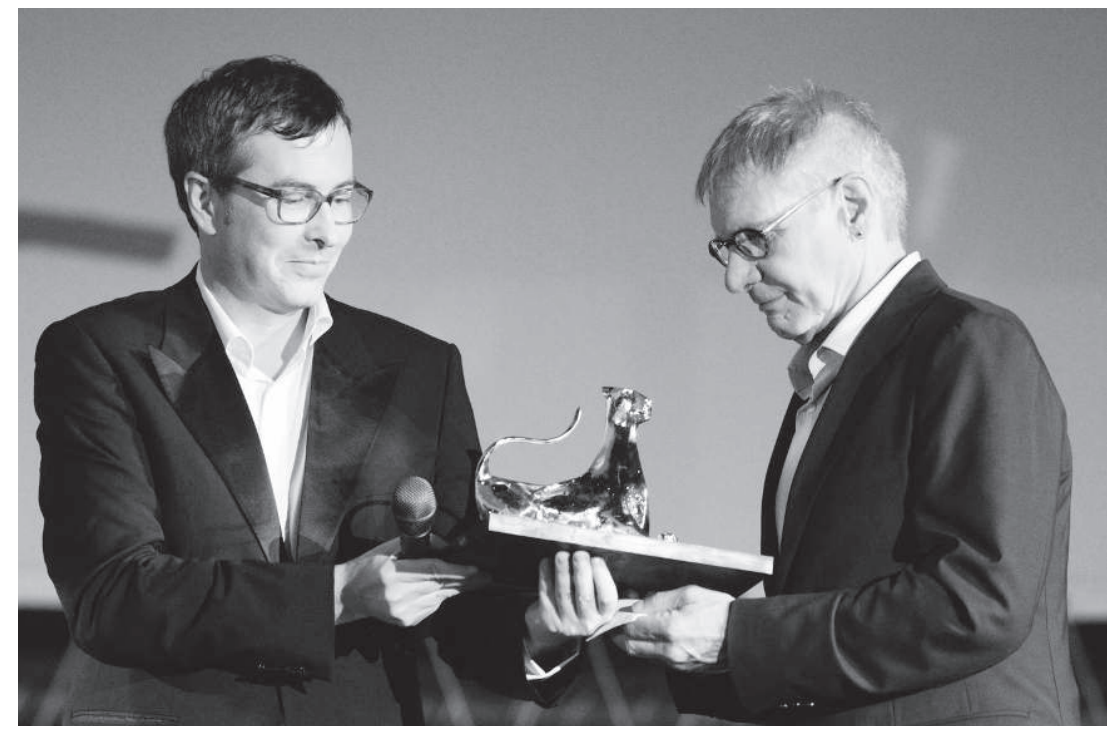


du cinéma, et le moyen le plus évident était alors de devenir réalisateur. Mais j’étais moins intéressé par le fait de faire des films que d'en voir.

\section{La Cinémathèque française a joué un rôle déterminant} dans votre parcours...

A Paris, où je passais tout mon temps libre dans les salles de cinéma, je fréquentais assidûment la Cinémathèque française; c'était l'époque de Dominique Païni 7, la programmation y était très intéressante. A force d'y être tous les soirs, je me suis lié d'amitié avec les gens qui y travaillaient: Dominique Païni justement, Jean-François Rauger et Philippe Arnaud à la programmation, ainsi que d'autres collaborateurs. C'est ainsi qu'ils m'ont proposé d'intégrer l'équipe. N'ayant pas fait mon service militaire, j’ai d'abord été embauché comme objecteur de conscience. Grâce à eux, j'ai appris le métier de programmateur et une certaine forme de théorisation de l'histoire du cinéma.

\section{Comment pourriez-vous définir ce métier de program-} mateur tel que vous l'avez appris à la Cinémathèque? Selon l'héritage d'Henri Langlois, il faut tout conserver: il est possible de tout montrer mais pas n'importe comment, suivant l'idée que la programmation n'est pas un acte créatif mais une forme de montage de l'histoire du cinéma qui consiste à associer les films les uns avec les autres, à créer des correspondances entre des œuvres de différentes époques. Il s'agissait également de montrer que la modernité traverse l'histoire du cinéma, du muet jusqu'à aujourd'hui, et que des auteurs très en avance à leur époque rejoignent la modernité, par exemple en associant Feuillade et Rivette, Vigo et Garrel. C'était un travail passionnant qui consistait, en somme, à organiser des rétrospectives, des cycles, des hommages en jouant de façon

7 Dominique Païni a été directeur de la Cinémathèque française de 1993 à 2000.

8 Le Festival de Locarno a proposé en 2010 lors de sa $63^{\mathrm{e}}$ édition la rétrospective intégrale des films d'Ernst Lubitsch qui a été reprise à la audacieuse et provocatrice avec l'histoire du cinéma. J'ai fait ça pendant une dizaine d'années avec énormément de plaisir, cela m'a ouvert au monde en rencontrant des critiques, des cinéastes et des cinéphiles. En fait, la cinéphilie est quelque chose de plutôt autarcique, une forme de protection face au monde et à la société : l'évasion y est mentale. L'opportunité d'en faire un métier ouvre le champ des relations humaines. Mes activités à la Cinémathèque m’ont permis de me faire repérer lorsqu'ils recherchaient un nouveau délégué général pour la Quinzaine des réalisateurs à Cannes: j’ai pu aménager mon emploi du temps pour travailler à Cannes et à la Cinémathèque.

A l'aune de votre parcours professionnel, évoquons maintenant le Festival de Locarno dont vous avez pris la direction artistique en 2010. Il semble qu'au cours de ces deux dernières éditions, on assiste à une évolution de la programmation qui tend à assimiler le festival à une cinématbèque, notamment dans le choix des rétrospectives ${ }^{8}$.

Je pense que ce sont deux choses très différentes. Pour un festival, au niveau des sections de la compétition, il faut compter avec qui a été produit dans l'année. Par ailleurs, la programmation d'un festival comme Locarno est différente de celle de la Quinzaine des réalisateurs puisque, pour cette dernière, il s'agit uniquement d'une sélection de vingt films. A Locarno, il s'agit à la fois de sélection et de programmation. En effet, les rétrospectives et les hommages que j'ai organisés lors des deux dernières éditions se calquent sur le modèle d'une cinémathèque et plus particulièrement sur le modèle de la Cinémathèque française - qui reste un modèle pour toutes les cinémathèques du monde ainsi que pour les festivals qui organisent des monographies. Le modèle de Langlois était de

Cinémathèque française et à la Cinémathèque suisse. En 2011, lors de sa $64^{\mathrm{e}}$ édition, il a programmé la rétrospective intégrale de Vincente Minnelli, également reprise à la Cinémathèque suisse. 
tout montrer, comme je l'ai dit auparavant: donc si on fait un hommage à Lang ou à Hitchcock, on montre tout sans déterminer ce qui est un bon ou un mauvais film.

\section{Quels sont les critères d'un tel choix? Est-ce la perspec- tive auteuriste qui le détermine?}

Non. Dans le souci d'exhaustivité, il s'agit de faire un travail d'historien : on donne à montrer le travail d'un cinéaste ou la production d'une époque, d'un pays. Mais le principe de la Cinémathèque française était de ne pas choisir, de ne pas sélectionner, et Langlois collectionnait, empilait, tout ce qu'il pouvait trouver. Tout montrer est essentiel, si on veut faire un travail, une étude sérieuse sur un auteur.

Précisément: mais cette tâche ne revient-elle pas plus à une Cinémathèque qu'à un festival?

La différence entre les deux réside dans la temporalité : tandis que la Cinémathèque peut construire une programmation sur de longues périodes durant toute l'année, sans interruption, le festival se déroule dans un temps restreint, dix jours en l'occurrence pour Locarno, et c'est un élément avec lequel il faut composer dans le choix d'une rétrospective intégrale. Je ne pourrais pas faire l'intégrale de John Ford, puisqu'il a fait plus de 100 films, mais lorsque je propose Ernst Lubitsch (2010) ou Vincente Minnelli (2011), je choisis des cinéastes dont je peux, au cours du festival, montrer tous les films.

\section{La rétrospective d'un festival tel que celui de Locarno} ne pourrait-elle pas être thématique et centrée sur une époque, une région ou un courant?

En effet, mais ce choix est lié à mon goût personnel et à ce que j'ai appris à la Cinémathèque française: c'est aussi l'enseignement que j'ai reçu auprès de JeanFrançois Rauger qui est plus attaché à l'idée de présenter la rétrospective d'un cinéaste qu'à célébrer un courant ou illustrer une époque. A cela s'ajoutent des raisons plus pragmatiques puisqu'un nom, un auteur, est plus identifiable qu'une thématique. Les journalistes vont comprendre, et par conséquent, il y aura plus de couverture de presse: le public va comprendre, et s'y rendre. Si tu fais quelque chose de plus compliqué comme la Nouvelle vague tchèque ou le Cinema Novo brésilien, ça va être plus difficile à vendre, donc ça va moins bien fonctionner. Dans les compétitions, parmi les films récents on ne va jamais sélectionner un film en pensant qu'il va faire beaucoup d'entrées, ce n'est pas du tout un critère! En revanche, dans la rétrospective et les hommages, l'idée est d'allier la qualité d'une ouvre à une dimension attractive pour le public. Le fait que la rétrospective ait bien marché a été très positif pour le festival et pour moi-même également. C'est normal que le festival subisse certaines attaques par rapport à la radicalité de la sélection, parce qu'il y a cet aspect de laboratoire que j'ai déjà mentionné, qui ne cherche évidemment pas à montrer des films commerciaux ou des films grand public: ce n'est pas le rôle d'un festival tel que celui de Locarno. Donc c'est bien de contrebalancer la programmation, pour maintenir l'équilibre, avec des hommages ou des rétrospectives qui plaisent à un autre public, plus traditionnel, plus "cinéphile à l'ancienne» qui va prendre du plaisir à Locarno. Le festival a cette particularité, contrairement à Cannes, de disposer de plusieurs espaces de projection qui correspondent à plusieurs publics très différents. Il faut donc sélectionner et programmer tout en équilibrant.

\section{Les rétrospectives sont organisées en collaboration avec les Cinémathèques française et suisse...}

Le Festival collabore avec les Cinémathèques, car elles ont à leur disposition beaucoup de copies, et l'idée est de montrer les films en $35 \mathrm{~mm}$, dans des copies de bonne qualité aux formats d'origine, et non pas de passer des DVD ou des Beta comme font certains festivals. 
Ily a donc un processus de délocalisation de Lausanne et de Paris vers Locarno?

$\mathrm{Si}$ je dirigeais un festival à Paris, je ne procéderais pas de la même façon. Il est vrai que l'idée est de délocaliser la programmation dans un lieu déterminé, Locarno, durant un moment particulier, ces dix jours d'été, où il y a beaucoup de spectateurs qui n'ont pas forcément accès à ces films. Si Paris Cinéma voulait faire un hommage à Minnelli, ils montreraient trois ou quatre films, mais certainement pas tout, car ce serait le rôle de la Cinémathèque ou de Beaubourg. Locarno, comme lieu, ne connait pas de concurrence, même avec Lausanne: on peut donc se permettre de délocaliser une proposition d'une Cinémathèque.

\section{Est-ce que ça répond à une demande du public?}

Je pense que le succès de la rétrospective Lubitsch ${ }^{9}$ l'a démontré. Contrairement à Cannes, Locarno a un public hétérogène, composé de professionnels, de personnes qui s'intéressent à l'aspect laboratoire du festival à travers les découvertes et les auteurs du cinéma contemporain, et de cinéphiles, qu'on pourrait appeler «les habitués», le public fidèle de Locarno, qui suit les rétrospectives. J'essaie de contenter tout le monde et de m'y retrouver moi aussi, parce que pour que je puisse proposer un programme qui fasse plaisir au public, il faut évidemment qu'il m'enthousiasme en premier lieu. Je choisis des auteurs qui me plaisent, que je me sens capable de défendre, dont j'arrive à faire partager l'enthousiasme.

\section{Vous participez ainsi à créer de la cinéphilie?}

Je ne sais pas, car je ne me fais pas beaucoup d'illusions, et j'ai pu constater que les rétrospectives sont suivies

9 En mars 2011, Olivier Père n'avait dirigé encore qu'une édition, la $63^{\mathrm{e}}$, où la rétrospective Ernst Lubitsch avait été présentée.

10 Créée en 1992 par la Cinémathèque française, la Cinémathèque. Revue semestrielle d'esthétique et d'histoire du cinéma, éditée par Dominique Païni et Marc Vernet, paraît de façon trimestrielle. par un public relativement âgé. Cela rentre dans une problématique plus générale: même pour les sections de la compétition, il y a très peu de jeunes gens et d'adolescents (peut-être un peu plus sur la Piazza). On constate un vieillissement de la cinéphilie. Cela s'explique par le fait que le cinéma est un art et un divertissement du $\mathrm{XX}^{\mathrm{e}}$ siècle, et que les spectateurs nés dans les années 1990 n'ont pas du tout le même rapport au cinéma, et particulièrementàla salle de cinéma, que ceux qui sont nés dans la deuxième moitié du $\mathrm{XX}^{\mathrm{e}}$ siècle.

\section{A Locarno, dans quelle section s'exprime le mieux la cinéphilie?}

Idéalement, dans toutes les sections, car il y a une cinéphilie très contemporaine qui consiste à être à l'affût des cinéastes importants aujourd'hui, qui ont du talent et qui sont les espoirs de demain. Mais cela dépend du mode de cinéphilie, pour certains le cinéma se termine en 1959 ou dans les années 1980. Moi, par exemple, je ne suis pas un spécialiste du cinéma muet ou du cinéma documentaire: c'est vrai que mon centre d'intérêt se concentre sur le cinéma des années 1930 jusqu'à aujourd'hui. Je ne suis pas sûr que si on faisait une rétrospective de films muets à Locarno, cela aurait le même succès que Lubitsch ou Minnelli. Le public est allé voir les films muets de Lubitsch parce que c'était Lubitsch.

La cinéphilie devrait ainsi s'exprimer dans toutes les sections: d'ailleurs ce serait un objectif, cela signifierait que les sections de la compétition sont suffisamment fortes pour attirer l'attention et susciter l'intérêt des cinéphiles. Il faut donc proposer des films récents suffisamment bons pour que les cinéphiles puissent s'y intéresser.

La cinéphilie est-elle liée au fait de produire des textes sur le cinéma, d'avoir vous-même une activité de journaliste, de critique?

Dominique Païni incitait ses collaborateurs, en plus de la programmation, à créer de la pensée autour des films en écrivant sur ceux-ci. Il avait d'ailleurs créé une revue: Cinémathèque ${ }^{\mathbf{1 0}}$, qui devait permettre de 
concrétiser cette volonté de proposer un accompagnement critique de l'histoire du cinéma mais aussi du cinéma contemporain. Ce fut pour moi le début de l'écriture : j'ai été influencé, aidé, motivé et poussé par ces personnes.

J'ai compris avec eux, à la Cinémathèque française, que pour avoir une certaine importance et surtout une certaine respectabilité dans le milieu de la cinéphilie, tu es obligé de passer par l'écriture. Tu peux très bien articuler des discours brillants à la sortie d'une projection à la Cinémathèque, mais il est vrai que seuls les écrits restent! Donc publier dans des quotidiens, des hebdomadaires, des revues, ou encore mieux publier des livres, c'est ce qui donne existence au cinéphile et à l'historien. C'était un effort à fournir: au départ, l'écriture n'était pas quelque chose d'évident, c'était douloureux et je n'y ai pas toujours pris beaucoup de plaisir, mais c'était important de le faire. Je tenais un carnet mais j’ai mis quelques années à franchir le cap et à écrire sur le cinéma : j’ai écrit quelques textes pour des fanzines mais je n'étais pas sûr de moi, je lisais Daney, Bazin, les grands critiques français et ça me donnait des complexes par rapport à ce que je pouvais produire et le sentiment qu'il n'était pas nécessaire d'en rajouter.

Puis on m'a commandé un article (sur le marché du film à Cannes) dans les Inrocks et c'est comme ça que j'ai commencé. Aujourd'hui, je continue à écrire de manière ponctuelle dans les Inrocks, les Cahiers $d u$ cinéma, j’ai coécrit un livre sur Jacques Demy et j’ai participé à un livre Phaiedon sur les 100 cinéastes de l'avenir.

\section{A qui s'adresse votre blog, placé sous l'égide du Festival de Locarno?}

Ça concerne les cinéphiles, la presse, les réalisateurs et les producteurs qui, un jour ou l'autre, ont la possibilité de montrer leur film à Locarno. Le blog constitue une forme de propagande. Même si le festival est un évènement ponctuel, il est important qu'il existe toute l'année, pour une question d'image, de visibi- lité. Cannes, tu en parles en février, tout le monde voit ce que c'est: pour Locarno, les gens ont tendance à l'oublier hors de la période du festival. Mon rôle est que les gens pensent à Locarno durant toute l'année d'une manière ou d'une autre.

\section{Pour ce faire, vous vous servez de la cinéphilie...}

Je ne m'en sers pas, mais c'est l'angle que je prends naturellement car ma relation au cinéma passe par la cinéphilie. Si j'étais une autre personne, je me servirais de ce blog pour parler de mes voyages, de mes dîners, de mes rencontres avec des gens du cinéma, sous un angle disons plus "mondain". Je pourrais le faire, ce serait amusant, mais moi j'applique plutôt la politique du secret: si la visibilité et la représentation sont importantes, le secret l'est également. On peut réussir à Locarno en préservant une certaine intimité avec les réalisateurs et les auteurs, sans étaler au grand jour nos relations. Il s'agit d'avancer masqué, notamment en raison de la concurrence avec les autres festivals.

La cinéphilie en France dans les années 1950 a permis de légitimer des auteurs américains considérés alors comme mineurs. Est-ce dans cet esprit que vous proposez lors du Festival de Locarno 2010 Step Brothers (Adam McKay, USA, 2008)11, une comédie américaine jouant sur le registre de la scatologie?

Il n'y a pas besoin d'être cinéphile pour apprécier Step Brothers et ce genre de films. Il s'agit d'une comédie qui fait rire avec un bon acteur comme John C. Reilly, à qui le festival rendait un bref hommage. Même si sur mon blog je ne m'épanche pas sur ma vie privée, mes sorties ou mes dîners en ville, je pense qu'à chaque fois qu'on programme un film ou qu'on écrit une critique, on dévoile une partie de soi-même si l'on est sincère. J'ai une culture très éclectique, et je n'ai

11 Le film est produit par Judd Apatow, spécialisé dans les comédies américaines au second degré. Les Cahiers du Cinéma lui ont consacré un numéro spécial en octobre 2009. 
aucun préjugé sur les films. Les comiques américains de la nouvelle génération sont souvent très bons, en particulier ceux qui s'inscrivent dans une tradition du comique héritée de Jerry Lewis, Dean Martin ou Blake Edward, c'est-à-dire un "moment» plutôt intéressant de la comédie américaine. Il y a toujours eu en France, à quelques exceptions près, un mépris et un sentiment de supériorité par rapport à la culture américaine émanant d'une certaine critique bourgeoise bien-pensante et de gauche. Télérama, qui considère les films d'horreur et les comédies comme des objets stupides auxquels il ne vaut pas la peine de s'intéresser, est emblématique de cette tendance. Et de l'autre côté, il y a un mouvement qu'on pourrait qualifier de snob, venu très tôt dans les années 1950, dans les Cabiers du Cinéma notamment, où au contraire on surévalue les objets mineurs et ingrats, on fait l'apologie de la série $\mathrm{B}$ ou de la série $\mathrm{Z}$, des films d'horreur, de la comédie. Et il y a des excès dans les deux sens.

Une certaine cinéphilie, comprise comme un besoin de réagir contre les classiques, privilégie, à certains égards, le décalage et la dérision mais implique également une perte du jugement esthétique par rapport à des films mineurs. N'est-ce pas l'effet que vous recherchez en proposant ce genre de films dans un festival comme Locarno? Pour ma part, j'ai toujours été curieux de découvrir ces films et mes goûts personnels entrent en jeu également. Et en effet, la dérision et l'humour font que j'aime beaucoup ce type d'objets a priori mineurs ou ingrats. Je prends l'humour très au sérieux.

Ce n'est d'ailleurs pas très éloigné de ce que j'ai fait en montrant L.A. Zombie (Bruce La Bruce, USA, 2010) en compétition internationale en 2010 : il y a là une part de provocation qui consiste à jeter un pavé dans la mare du festival, mais aussi la volonté de le considérer vraiment comme un objet d'étude qui m'intéresse beaucoup et pas du tout pour des raisons militantes gay (comme cela a été mentionné dans la presse, $n d l r$ ). C'est un objet passionnant pour étudier ce que le cinéma peut avoir de militant, non pas tant sur le plan de la revendication des minorités sexuelles que sur l'idée de liberté absolue qui permet de faire un film avec presque rien, sans argent, sans autorisation, en jouant, évidemment, avec le cinéma de genre comme le film d'horreur, le gore, etc. Je milite pour la présence de la comédie dans les festivals, et, d'ailleurs, la critique commence à prendre ça au sérieux, comme on a pu le voir avec les frères Farrelly et Judd Apatow. Les grands festivals n'ont jamais osé les suivre, ils en ont peur: les producteurs et les studios qui font ce type de films ne veulent pas aller dans les festivals car ils ont peur de la critique, tout comme les festivals d'ailleurs qui savent que le film ne va pas être pris au sérieux et que le jury ne va jamais oser donner le prix à ce genre de films. D’ailleurs, j'ai passé Cyrus (Jay Duplass, Mark Duplass, USA, 2010) sur la Piazza en $2010 \ldots$ 